\title{
Contrarreferenciamento farmacoterapêutico: experiências vivenciadas por profissionais de saúde em um hospital de ensino
}

\author{
Pharmaceutical counter-referencing in the transition care process: experiences of health \\ professionals in a teaching hospital
}

\section{Contra referencia farmacoterapéutica: experiencias vividas por profesionales de la salud en un hospital universitario}

\section{RESUMO}

Objetivo: Compreender o processo de contrarreferenciamento farmacoterapêutico na rede de cuidados, a partir das experiências de profissionais de saúde. Método: Estudo qualitativo, fundamentado na sociologia compreensiva, desenvolvido em um hospital de ensino, sendo entrevistados 14 profissionais de saúde. A coleta de dados ocorreu, por meio de entrevistas, realizadas a partir de um roteiro semiestruturado. Realizou-se análise de conteúdo e identificação de categorias. Resultados: Identificaram-se as categorias os "des"caminhos do referenciamento e em busca de um contrarreferenciamento possível, em que o contrarreferenciamento apresenta-se como uma ação frágil, influenciado pelo medo da reinternação, questões de acesso e, principalmente, fragilidade dos processos de comunicação. Quanto às possibilidades de melhoria, têm-se preparação da alta com antecedência, reconhecimento das subjetividades dos pacientes e fortalecimento da comunicação. Considerações finais: O contrarreferenciamento é construído a partir das relações e vivências dos atores sociais, sendo que ocasiona impacto no processo de cuidado, devendo ser fortalecido.

Descritores: Assistência Hospitalar. Uso de Medicamentos; Continuidade da Assistência ao Pacient; Assistência à Saúde; Acesso aos Serviços de Saúde; Enfermagem.

\begin{abstract}
Objective: Understanding the perception that professionals about the process of pharmaceutical referencing of patients in the health system. Methodology: Case study based on a qualitative approach. The setting was a general teaching hospital, and the subjects were 14 health professionals. The data collection were done through interviews guided by a semi structured script. Results: The referencing was heterogeneous, taking into account relationships and subjectivities; it caused feelings of fear about readmission, pharmacotherapy unavailability, possible inability for the patient to purchase the drugs, and the fragility of communication. The professionals also pointed out possibilities to improve referencing such as to prepare a patient for discharge well in advance, implement referencing and recognition of patients' subjectivity mechanisms. Conclusion: referencing is built from the relationships and experiences of those involved. It is an action that impacts the care process and can be strengthened in the institution chosen for this study. Descriptors: Hospital Care; Drug Utilization; Continuity of Patient Care; Transition Care; Delivery of Health Care; Health Services Accessibility; Nursing.
\end{abstract}

\section{RESUMEN}

Objetivo: Comprender el proceso de contrarreferencia farmacoterapéutica en la red de asistencia médica Método: Estudio cualitativo, desarrollado en un hospital universitario, con 14 profesionales de la salud. La recopilación de datos se realizó por medio de entrevistas, realizadas con un guión semiestruturado. Resultado: Se identificaron las categorías, los "des"caminos de referencia y en busca de una posible contrarreferencia, donde la contrarreferencia se presenta como una acción frágil, influenciada por el miedo a la readmisión, en asuntos de acceso y en la fragilidad en los procesos de comunicación. En cuanto a las posibilidades de mejora, existe la preparación previa del alta, el reconocimiento de las subjetividades de los pacientes y el fortalecimiento de la comunicación. Consideraciones finales: La contrarreferencia se construye a partir de las relaciones y experiencias de los actores sociales, lo que tiene un impacto en el proceso de atención médica, y debe ser fortalecido.

Descriptores: Atención Hospitalaria; Utilización de Medicamentos; Continuidad de la Atención al Paciente; Prestación de Atención de Salud; Accesibilidad a los Servicios de Salud; Enfermería.

\begin{tabular}{|}
$\begin{array}{r}\text { Josiane Moreira da Costa } \\
0 \text { 0000-0001-6097-6994 }\end{array}$ \\
Kênia Lara Silva ${ }^{1}$ \\
$0000-0003-3924-2122$ \\
1 Universidade Federal de Minas \\
Gerais.
\end{tabular}

$\begin{array}{r}\text { Autor correspondente: } \\ \text { Josiane Moreira da Costa } \\ \text { E-mail: josycostta2@yahoo.com.br } \\ \hline\end{array}$

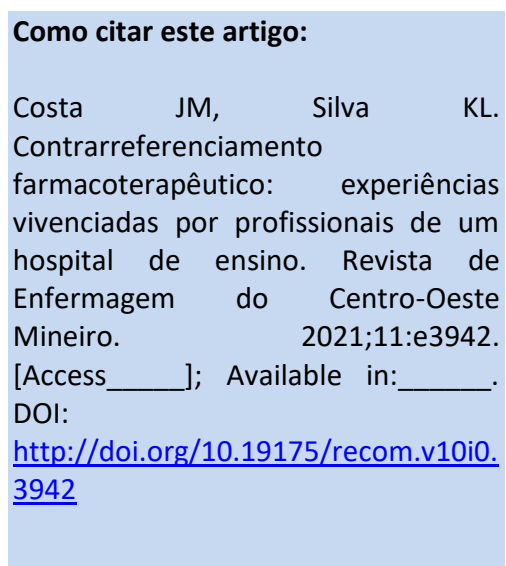




\section{INTRODUÇÃO}

Ao transitar na rede de cuidados, o paciente se relaciona com diferentes atores e serviços, que representam as diferentes estações de cuidado em saúde. Esse processo torna o caminhar na rede heterogêneo, pois envolve diferentes subjetividades entre os sujeitos que buscam e ofertam o cuidado em saúde ${ }^{(1)}$. É nesse contexto que se entendem a importância e os desafios existentes, para consolidar a integralidade do cuidado, entendida como o conjunto de aspectos que assegurem o direito do acesso dos usuários aos diferentes serviços oferecidos pelos diferentes níveis de atenção(2).

Além das diferentes subjetividades que permeiam o cuidar em saúde, entende-se que, ao caminhar na rede de cuidados, entre os diferentes níveis de atenção, os pacientes necessitam que esses níveis se articulem de forma que se garanta o oferecimento do cuidado de forma integral. Falhas nessa articulação podem ocasionar em agravos no estado de saúde dos pacientes. Os principais erros relacionados aos cuidados em saúde ocorrem quando um paciente se move de um serviço de saúde para outro(3), sendo o principal elemento para a integração das redes de saúde, o efetivo sistema de referência e contrarreferência.

A referência ocorre, quando um paciente é encaminhado de um determinado nível de atenção para outro mais complexo, sendo a contrarreferência o encaminhamento do paciente de um nível mais complexo para o de menor complexidade. Esses processos são definidos pelo Ministério da Saúde como um dos principais elementos de reorganização das práticas de trabalho em saúde no Sistema Único de Saúde(SUS) $)^{(4)}$. Cada nível de atenção possui especificidades que influenciam os processos de referência e contrarreferência, destacando-se o nível terciário. Esse é caracterizado por intensas intervenções e uso de tecnologias duras que, normalmente, exigem intensas modificações no processo de cuidado após a alta hospitalar ${ }^{(3)}$.

Em razão da polifarmácia, complexidade clínica dos pacientes atendidos e considerável número de intervenções, a hospitalização é uma situação que pode ocasionar importantes modificações no uso de medicamentos pelos pacientes, sobretudo, quando se consideram mudanças nas prescrições após a alta hospitalar ${ }^{(3)}$. Entende-se que os profissionais de saúde das instituições hospitalares possuem importante função, quanto ao uso de medicamentosos, durante e após a internação, pois eles são quem fazem a escolha da farmacoterapia, no período da internação, realizam o encaminhamento dos pacientes após a alta, são responsáveis pelo fornecimento de informações em relação aos medicamentos em uso e forma de acessá-los.

Erros de medicação decorrentes de prescrições inadequadas, que são comuns no ambiente hospitalar ${ }^{(5)}$, podem não ser identificados no momento da realização da prescrição de alta e continuar ocorrendo no âmbito da atenção primária ${ }^{(3,6)}$. Dessa forma, a internação poderia evidenciar-se como uma oportunidade de fortalecimento da autonomia do paciente, de modo que, no processo de contrarreferenciamento, os profissionais da Atenção Primária à Saúde participariam na discussão de condutas terapêuticas( ${ }^{(7)}$, fato não fortemente evidenciado na prática.

No início da proposição da pesquisa, observou-se que a Assistência Farmacêutica no Brasil era composta por diferentes programas que contemplam o fornecimento e estímulo ao uso racional de medicamentos nos âmbitos da Atenção Básica, Ambulatorial, e Hospitalar ${ }^{(8)}$. Entetanto não se verificava a existência formal de um processo de referência-contrarreferência que possuía como enfoque a farmacoterapia ${ }^{(5,6,8)}$. Apesar da literatura também apontar para as ações de cuidado farmacoterapêutico, esses eram relatados como ações isoladas, sem um referenciamento/contrarreferenciamento dos pacientes na rede de cuidados.

Quanto às questões da acessibilidade e importância do hospital, para a manutenção da continuidade da terapia medicamentosa, na transição de cuidado, associado à escassez de estudos brasileiros que contemplem essa temática, a presente pesquisa propõe a compreensão do contrarreferenciamento farmacoterapêutico, após a alta hospitalar, a partir das experiências de profissionais de um hospital de ensino.

\section{MÉTODO}

Trata-se de um estudo de caso com caráter qualitativo, sendo que compete à pesquisa qualitativa o estudo do universo da experiência vivida, sendo requerida a análise e interpretação dos significados e vivências das pessoas, além dos fenômenos ocorridos em um determinado grupo de pessoas. 
Utilizou-se como referencial teórico metodológico a sociologia compreensiva. Nessa teoria, deve-se considerar um conhecimento empírico cotidiano, com a valorização da socialidade. Propõe-se que o imaginário é algo que vai além do indivíduo e possui influência sobre o coletivo. $O$ entendimento das vivências ocorre, a partir da visão de diferentes atores, em relação a um determinado fenômeno denominado, neste estudo, contrarreferenciamento farmacoterapêutico. A interpretação de cada vivência considera as complexas relações sociais, em que cada fenômeno analisado é um sintoma do vivido no âmbito social ${ }^{(9)}$.

O cenário da pesquisa é um hospital geral de ensino, localizado na região Norte de Belo Horizonte, que possui 320 leitos e gestão informatizada por prontuário eletrônico. A clínica médica da instituição constitui objeto deste estudo, sendo composta por 96 leitos e uma equipe composta pelos seguintes profissionais que exercem atividades diretamente ligadas aos medicamentos: 16 enfermeiros, 8 farmacêuticos, 1 (um) residente em farmácia que exercia atividades clínicas, 64 técnicos de enfermagem e 18 médicos.

$\mathrm{Na}$ coleta de dados, realizaram-se entrevistas com roteiro semiestruturado, que foram elaboradas, a partir da pergunta norteadora que se pretendia estudar: "como os profissionais de saúde vivenciam o contrarreferenciamento farmacoterapêutico, tendo em vista as suas experiências em um hospital de ensino? Além disso, após imersão em leitura teórica sobre referência/contrarreferência e teoria fundamentadora do presente estudo, a pesquisadora responsável desenvolveu o roteiro, que foi enviado a duas pesquisadoras com experiência na temática que o aprovaram. Foram realizadas perguntas aos profissionais de saúde relacionadas à percepção da alta hospitalar e ao contrarreferenciamento dos pacientes na rede.

Os profissionais foram incluídos, conforme os seguintes critérios: possuir vínculo institucional por um período superior a três meses, exercer atividades no setor Clínica Médica da instituição e participar diretamente dos cuidados farmacológicos de pacientes idosos previamente acompanhados na Unidade Básica de Saúde de referência e que receberam alta hospitalar com prescrição medicamentosa. A escolha do período de três meses de vínculo institucional foi relacionado ao cumprimento do período de experiência empregatício, tempo que sugere um mínimo de contato necessário com as funções a serem desempenhadas e com o ambiente hospitalar. Já em relação à Clínica Médica, esse setor foi escolhido por ser referência no atendimento de pacientes com comprometimento dos problemas crônicos de saúde, que necessitam utilizar de forma frequente os serviços de saúde.

Os pacientes foram identificados, por meio de relatório informatizado de gestão, referente ao setor Clínica Médica, identificando-se aqueles que tiveram alta hospitalar no período de um mês anterior ao início da coleta de dados. Em seguida identificaram-se, em prontuário eletrônico, registros de acompanhamento prévio pela Atenção Primária, prescrição de medicamentos no momento da alta hospitalar e recomendação de retorno ambulatorial no ambulatório de anticoagulação da instituição.

Em um mês de análise, identificou-se alta de 20 pacientes que correspondiam aos critérios de inclusão. A partir dessa identificação, foi emitido um segundo relatório que continha os nomes de todos os profissionais que exerceram cuidados medicamentos diretos a esses pacientes, durante a internação, sendo consideradas os profissionais médicos, enfermeiros, técnicos em enfermagem e farmacêuticos.

Em um primeiro momento, foi selecionado um profissional de cada categoria, para participação na pesquisa, sendo que, para cada categoria, identificou-se o profissional com maior tempo de casa. Caso o profissional recusasse em participar da pesquisa, o profissional com o segundo maior tempo de casa era convidado a participar no estudo.

No final da realização das entrevistas com um representante de cada categoria profissional, iniciou-se um novo ciclo de captação de profissionais e entrevistas, até a ocorrência de saturação dos dados. Ressalta-se que, após a definição dos profissionais a serem entrevistados, o convite era realizado a todos, em um mesmo dia, sendo a disponibilidade de horário de cada profissional o critério para a definição da ordem das entrevistas.

Os profissionais foram convidados a participar na pesquisa nos respectivos setores de trabalho, sendo que as entrevistas foram realizadas, nos horários de descanso ou em horários fora do expediente, mediante a disponibilidade de cada profissional e em local que garantia a privacidade. 
Após a gravação de cada entrevista, o arquivo recebia uma codificação correspondente ao participante e, em seguida, era salvo em programa informatizado. No processo de codificação, cada entrevistado recebeu as iniciais de sua categoria profissional, sendo gerada uma aleatorização no programa excel para a escolha numérica de cada entrevistado. Dessa forma, a codificação era composta por iniciais da categoria, seguida de um número aleatorizado. Para facilitar a identificação da saturação, as primeiras entrevistas foram transcritas, logo após a sua realização, considerando-se os limites empíricos do objeto analisado e a sensibilidade e aprofundamento do pesquisador em relação ao tema pesquisado. A saturação foi reconhecida, ao se identificar a ocorrência da realização de três entrevistas de forma consecutiva, nas quais não se identificou nenhum elemento novo dos relatos previamente realizados e analisados ${ }^{(10)}$.

Após a finalização do processo de coleta de dados e a transcrição de todas as entrevistas, os dados foram submetidos à análise de conteúdo. Para isso, seguiram-se as etapas 1) ordenação dos dados, por meio de leituras exaustivas dos relatos, para estabelecer um sentido ao conjunto de proposições; 2) leituras sistematizadas com o objetivo de encontrar semelhanças ou contradições nos relatos, organizando os achados em "unidades de significados"; 3) organização das unidades de significados em temas, buscando um aprofundamento do conteúdo das mensagens; 4) interpretação dos temas e discussão com a literatura existente; 5) elaboração de relatório final com a interpretação realizada, sabendo que "o produto final é sempre provisório"(10).
Cada entrevista teve durabilidade média de 40 minutos, sendo que a coleta dos dados teve durabilidade de um mês, sendo os resultados incorporados ao projeto de mestrado intitulado: "acesso à terapia medicamentosa na perspectiva de pacientes e profissionais de saúde", da Escola de Enfermagem da UFMG. Os dados foram validados pelos próprios participantes, de modo que o resultado foi apresentado a eles antes da publicação. O relato de eles se sentirem representados, ao lerem as falas e respectivas interpretações, foi considerado uma validação.

Ao serem contatados, os participantes receberam informações sobre a pesquisa, sendo que, nesse processo, foi apresentado o termo de consentimento livre e esclarecido (TCLE), que especificava sobre a garantia do anonimato, necessidade da gravação das entrevistas. Àqueles que consentiram na participação, solicitou-se a assinatura do TCLE. A realização da pesquisa ficou a cargo de uma pesquisadora previamente treinada, que trabalhava na instituição mas não exercia cuidado direto aos pacientes.

O presente estudo foi submetido à avaliação do Núcleo de Ensino e Pesquisa (NEP) do Hospital em estudo e ao Comitê de Ética em Pesquisa (COEP) da UFMG, recebendo o parecer número 00330203000-11.

\section{RESULTADOS E DISCUSSÃO}

Determinaram-se, após a saturação, 14 profissionais, sendo três enfermeiros, três farmacêuticos, uma residente em farmácia, quatro médicos e três técnicos em enfermagem, conforme verificado na Tabela 1.

Tabela 1 - Perfil dos profissionais que participaram da pesquisa.

\begin{tabular}{cccc}
\hline № profissional & Profissão & Tempo de trabalho no Hospital & Sexo \\
\hline 1 & Enfermeiro & 5 anos & Masculino \\
2 & Farmacêutica & 1 ano & Feminino \\
3 & Enfermeira & 1 ano & Feminino \\
4 & Residente de Farmácia & 7 meses & Feminino \\
5 & Técnico em Enfermagem & 4 anos & Masculino \\
6 & Enfermeira & 2 anos & Feminino \\
7 & Farmacêutica & 1,5 ano & Feminino \\
8 & Farmacêutica & 1 ano & Feminino \\
9 & Técnica em Enfermagem & 8 meses & Feminino \\
10 & Médico & 5 anos & Masculino \\
11 & Médica & 5 anos & Feminino \\
13 & Médico & 5 anos & Masculino \\
14 & Médica & 5 anos & Feminino \\
\hline
\end{tabular}

Fonte: Os autores, 2016. 
Após a análise das entrevistas identificaramse duas categorias denominadas Os "des"caminhos do referenciamento e Em busca de um referenciamento possível. O detalhamento dos achados encontra-se a seguir.

\section{Categoria 1: os "des" caminhos do contrarreferenciamento}

Nessa categoria, os entrevistados apresentaram 0 processo de contrarreferenciamento farmacoterapêutico no cotidiano do trabalho, sendo apresentados, além dos caminhos pelos quais 0 contrarreferenciamento acontece, os "des"caminhos que contribuem para a fragmentação.

Durante a internação, qualquer profissional que entra em contato com os pacientes é responsável pelo levantamento de informações sobre a farmacoterapia - como tipo de medicamento, horário, possíveis efeitos, se há necessidade de mudança ou de nova adequação. Há, inclusive, um trabalho contínuo com os da enfermagem em relação a uma prática informada sobre as ações desenvolvidas junto ao paciente: " questão da alta hoje, todos os pacientes que recebem alta são informados pelo corpo clínico de todas as medicações que eles vão fazer uso da alta hospitalar em diante. São feitas orientações para todos os pacientes (ENO1)". Entretanto os profissionais também reconhecem que essa ação não ocorre de forma contínua: "Quando se tem uma demanda e há pergunta da medicação, a gente pega e informa o que vai ser tomado, porque tá utilizando esse ou aquela medicação. Agora, quando o paciente não se interessa em perguntar o que é, acaba que, se ele tá interessado em saber, você tem que falar com ele" (ME10).

Ao afirmarem que o fornecimento de informações sobre a farmacoterapia no âmbito hospitalar ocorre mediante questionamento dos pacientes, os profissionais assumem que essa não é uma prática constante. Embora reconheçam que a maioria dos pacientes use os medicamentos sem saber por que, o ato de informar-lhes não é intrínseco às ações cotidianas. Identifica-se um paciente assujeitado, sem histórias, valores e percepções próprios, contrariando, inclusive, a ideia de que os pacientes podem ser mais bem informados sobre o uso eficaz de medicamentos por profissionais de saúde ${ }^{(11)}$. Isso também evidencia que as práticas de empoderamento não são inerentes às atuações profissionais(12): "Acho que aqui no hospital ele fica mais passivo mesmo, não se preocupa tanto [...], tem vários profissionais cuidando dele então acho que a preocupação dele em relação ao medicamento é menor!" (FA04).

Em alguns casos, os pacientes recebem orientações apenas sobre os medicamentos prescritos na alta. Mas a falta de informação e a adesão do profissional médico aos medicamentos disponíveis na Relação Municipal de Medicamentos (REMUME) do município, ao qual o paciente pertence, também, são consideradas barreiras:

"Depende do médico. Eu acho que alguns têm essa consciência do que tem na rede, do que ele vai dar pro paciente. Agora existem médicos também que não vão alterar e que ele [paciente] vai sair daqui com uma prescrição que ele não vai conseguir!(FA02) Se não tiver no posto de saúde aí a família tem que adquirir a medicação, que aí é o que é o difícil!" (ENO6).

No momento da alta, ao não prescrever os medicamentos fornecidos pelos municípios, o médico acaba por colocar barreiras que poderão comprometer $O$ acesso. E mesmo com investimentos do governo em programas para a melhoria do acesso a medicamentos, como os Aqui tem Farmácia Popular e Farmácia popular do Brasil $^{(13)}$, esse ainda continua sendo um desafio ${ }^{(14-}$ 15). Seria necessária maior apropriação, em nível hospitalar, sobre os medicamentos disponíveis na rede e sobre as possibilidades do paciente em adquiri-los, para que a prescrição seja acessível.

Eles também relatam que falhas no processo de contrarreferenciamento comprometem a continuidade do cuidado e, consequentemente, a farmacoterapia:"É [...] a gente não tem essa questão da referência e contrarreferência, então é um tiro no escuro mesmo! Porque a gente sabe que às vezes é difícil o acesso!" (ME11).

Também entende-se que esses fatores podem contribuir para que a reinternação seja um acontecimento provável: "Ele vai voltar pro hospital! (ME12). Quando acaba o remédio não tem como comprar e ele fica sem. Aí então descontrola! Uma ICC que vinha bem, fica uma semana sem diurético, acabou! Aí ele vai internar aqui. Aí ele fica mais 10, 15 dias no hospital" (ME14).

Além da garantia do acesso, é necessária a garantia do acesso de forma integral, sendo que a defesa do acesso igualitário, num sistema de saúde universal, assume a forma da defesa ao acesso a todos os níveis de atenção por um mesmo 
paciente. A existência de articulação e contrarreferenciamento seguro, mostra-se, ainda, dependente do conhecimento da acessibilidade ampla, considerando as necessidades do sujeito e as tecnologias realmente acessíveis e que deverão ser utilizadas caso a caso. 0 contrarreferenciamento, sem o conhecimento do todo, apresenta-se incompleto e frágil e pode não alcançar os objetivos propostos.

Em virtude da necessidade da manutenção do oferecimento de cuidados específicos, no processo de transição entre os níveis de atenção, os profissionais reconhecem na alta hospitalar um momento de imprevisibilidade: "Eu acho que ficam muito perdidos. Eu costumo falar com as meninas e com meus colegas que, quando eu dou alta pro paciente, a impressão que eu tenho é que é um passarinho que tá saindo do ninho pela primeira vez! [...] Ele até vai receber uma dose quando ele sair daqui suficiente pra manter o tratamento mais um tempo, mas depois ele vai conseguir isso?" (ME12). " Imagina um paciente que não é da área de saúde, que não entende, que não sabe "pra quê" "(ENO1).

A descontinuidade da farmacoterapia também se caracteriza pela dificuldade em adquirir os medicamentos prescritos fornecidos pelo SUS e pela incompreensão por parte dos usuários da maneira como utilizá-los. Para os entrevistados, é como se o paciente se sentisse "perdido" na rede, em relação aos caminhos a serem percorridos, no âmbito da assistência farmacêutica, sendo que a prescrição e a dispensação têm que envolver informações que, muitas vezes, vão além do prescrito e, por isso, necessitam de ações educativas e acompanhamento contínuo ${ }^{(16)}$. Nesse contexto, ocorre um processo cíclico, em que o período pós-alta pode desencadear futuras reinternações, o que faz dele um processo "póspré (re)internação hospitalar" : "...se não tiver no posto de saúde, aí a família tem que adquirir a medicação, que aí é o que é o difícil! Porque os nossos pacientes a maioria são carentes, então a maioria não tem condição" (EN06).

Um estudo que avalia readmissões hospitalares identificou que problemas de adesão estavam dentre as principais causas evitáveis de readmissões hospitalares ${ }^{(17)}$. Isso reforça o fato de que, além da disponibilidade, é necessário que a dose seja adequada para o paciente e que ele entenda, queira e consiga utilizar os medicamentos ${ }^{(18)}$. Informações incompletas ou imprecisas, no período da hospitalização, podem contribuir para deficiências no processo de tomada de decisões nos demais níveis de cuidado(6). Também identifica-se que os problemas do contrarreferenciamento, considerados de "âmbito externo" pelos entrevistados, acabam por refletir as limitações "internas" do contrarreferenciamento, pautadas na dificuldade dos profissionais do hospital de construírem um olhar único sobre o paciente-sujeito e sobre a farmacoterapia.

\section{Categoria 2: em busca de um contrarreferenciamento possível.}

$\mathrm{Na}$ categoria contrarreferenciamento possível, os entrevistados também apontam as possíveis estratégias para facilitar o processo de referência e contrarreferência na atenção à saúde.

Preparação prévia da alta hospitalar e fortalecimento do processo de comunicação são algumas das propostas indicadas: " Então assim a gente observa que a farmácia faz tudo muito explicadinho, tipo assim: "você vai tomar isso aqui 10 horas, isso meio dia, isso tal hora", tudo muito explicadinho!" (EN03).

A preparação prévia da alta, identificação de especificidades dos pacientes e familiares, em relação ao uso dos medicamentos, capacitação técnica dos profissionais, apresentam-se como ações de fortalecimento da informação, entendida neste estudo de forma mais ampla, ou seja, no sentido de conhecimento, participação, comunicação. Também pode-se inferir que há um pensamento de rede de atenção verticalizada, quando profissionais recomendam o uso de medicamentos não disponibilizados pela atenção primária.

Em caso de necessidade de uso de medicamentos não disponibilizados pelo SUS, o entendimento das limitações socioeconômicas dos pacientes influencia uma prescrição que possa ser acessivel, por meio da negociação com os familiares: "Pra ele ser informado, durante todo o processo e até em toda rede mesmo, quando ele sair. Não só entregar, tá, "tem captopril pra você, ótimo!" Não! Tem que ter um acompanhamento na rede, no posto de informação, um acompanhamento dele lá fora"(FA02).

A informação contínua propiciaria ações que aguardam sair do papel para se tornarem uma prática cotidiana: "E eu tenho encaminhado todos os pacientes pra fazer controle no posto de saúde pra ver se diminui esse problema de [...](ME10) Então eu acho que talvez podia melhorar a 
orientação da alta pra alguns grupos! Mas eu acho que aqui tem que caminhar pra isso!" (ME11).

É necessário um trabalho em equipe que deve ser entendido como um conjunto de práticas individuais, ações e relações entre os níveis assistenciais, que requer um cuidado em saúde longitudinal, em uma linha de mão dupla que transpassa a atenção primária em direção ao setor terciário e assim retorna ${ }^{(19)}$.

A prática sistemática de avaliação dos encaminhamentos para o centro de saúde poderia melhorar a qualificação das referências e promover utilização mais racional dos recursos especializados. Esforços deveriam ser empreendidos, quanto à necessidade de garantir a continuidade informacional, requisito para a coordenação do cuidado pela Atenção Primária à Saúde ${ }^{(20)}$.

Extrapola-se, portanto que, para se pensar uma acessibilidade possível, é preciso reconhecer a necessidade de adequação de nossas ofertas ao contexto específico da situação no qual se dá o encontro do sujeito com a equipe de saúde ${ }^{(21)}$. Experiências internacionais de implementação de serviços com esse enfoque demonstraram contribuições para a prevenção de erros de medicação, complicações clínicas e reinternações ${ }^{(22-24)}$.

O fortalecimento da comunicação, entretanto não deve ser apenas entre profissionalpaciente-unidades, mas também entre os profissionais da instituição: "A gente recorre à enfermeira, que recorre ao médico e procura saber se tá certo, se a dose tá certa. Assim quando a gente não sabe, a gente pergunta o enfermeiro pra ele nos informar qual é, pra que o remédio serve, aí se ele não sabe ele procura o médico" (TE05).

O fortalecimento da comunicação não deve ser apenas entre profissional paciente, mas deveria ocorrer, também, entre profissionais, para que haja melhor entendimento em relação aonde e a quem está se referenciando o paciente, que até a alta estava sob os seus cuidados ${ }^{(25)}$. Orientações devem ser dadas a qualquer momento, em qualquer nível e por qualquer profissional, para se evitarem falhas nos diferentes processos de trabalho em saúde.

Reafirma-se a informação como uma estratégia fundamental para o acesso. Deve, também, ser em rede e contínua, o que certamente facilitará o caminhar do usuário, sendo que há necessidade de aumentar esforços para assegurar que trocas efetivas de informações, no processo de transição do cuidado, sejam apontadas pela literatura ${ }^{(1)}$.

Mediante a necessidade do fortalecimento da comunicação interprofissional, conhecimento e uniformização dos processos de trabalho e acompanhamento da alta rotatividade de profissionais, o treinamento e ou capacitação é colocado como uma das possíveis estratégias que poderiam contribuir para a manutenção do acesso durante a internação: "Algum conhecimento bem amplo a gente tem, mas tem alguns ainda que a gente tem dificuldade pra que serve, qual é a função, se pode ser associado a outras medicações" (TE05). "Então eu acho que assim antigamente a gente tinha esses cursinhos [sobre medicamentos], mas depois parou" (TE13).

Os profissionais relataram que 0 investimento em capacitação interna na administração de medicamentos é uma das estratégias, para amenizar as dificuldades dos técnicos de enfermagem, em relação aos medicamentos e, assim, evitar possíveis erros. Sobre a necessidade de capacitação dos profissionais de saúde, para atuarem na transição do cuidado, deve ser direcionada a profissionais de diferentes âmbitos: desde a graduação até a pósgraduação(26).

Entende-se que essas ações gerariam prescrições mais acessíveis e relatórios de alta melhor pautados na continuidade do cuidado, mais condizentes com a realidade de vida diária dos pacientes, o que traria contribuições para os pacientes atendidos.

\section{CONCLUSÃO}

O contrarreferenciamento farmacoterapêutico apresenta-se constituído de fragilidades, devendo ser melhor fortalecido na instituição em estudo.

A inexistência de um processo de comunicação não consolidado entre os profissionais de saúde e entre profissionais e pacientes contribui para o enfraquecimento de ações isoladas referentes à identificação dos medicamentos utilizados no período préinternação, ao fornecimento de informações durante a internação e, para o próprio processo de contrarreferência dos pacientes na rede, o que propicia falhas no processo de cuidado farmacoterapêutico.

Apesar de existentes, as ações que contribuem para um melhor processo de referenciamento são realizadas, a partir de 
iniciativas individuais, sendo que a implementação de uma política institucional poderia contribuir para o fortalecimento desse processo.

Reafirma-se, então, a necessidade de o referenciamento e o contrarreferenciamento tornarem-se realmente uma estratégia de comunicação e integração entre os profissionaisusuários e profissionais-serviços de saúde, para a busca de melhorias.

Acredita-se que os achados possam contribuir para o aprimoramento e processo de sistematização das práticas de enfermagem relacionadas ao referenciamento e contrarreferenciamento dos paciente na rede de cuidados. Além disso, as características e vivências dos profissionais, em relação ao contrarreferenciamento farmacoterapêutico, apontam para a necessidade multiprofissional de condução desse processo, que é permeado por diferentes saberes. A elaboração de formulário de contrarreferenciamento multiprofissional, no qual estivessem especificados os medicamentos utilizados, durante a internação e prescritos na alta, informações subjetivas dos pacientes que refletissem as suas experiências medicamentosas, assim como informações sobre o acesso, poderiam contribuir para o fortalecimento do contrarreferenciamento com consequente melhoria na integralidade do cuidado.

Este estudo possui a limitação de refletir a vivência de profissionais vinculados a uma instituição de saúde, sendo que outra realidade pode ocorrer em diferentes contextos.

Espera-se que as estratégias apresentadas, entre outras a serem pensadas, facilitem o acesso à farmacoterapia com continuidade do cuidado de forma longitudinal na rede de atenção.

\section{REFERÊNCIAS}

1 - Jeffs L, Saragosa M, Law M, Kuluski K, Espin S, Merkley J. Elucidating the information exchange during interfacility care transitions: Insights from a qualitative study. BMJ Open 2017;7:1-7. DOI: 10.1136/bmjopen-2016-015400

2 - Giovanella L, Lobato LVC, Carvalho Al, Conill EM, Cunha EMDA. Sistemas municipais de saúde e a diretriz da integralidade da atenção: Critérios para a avaliação. Saúde Debate 2003 [citado em 15 mar 2020]; 26(60):37-61. Acesso em: http://www6.ensp.fiocruz.br/repositorio/sites/def ault/files/arquivos/SistemasMunicipaisSa\%C3\%BA de.pdf
3 - Smith L, Mosley J, Lott S, Cyr Júnior E, Amin R, Everton $E$, et al. Impact of pharmacy-led medication reconciliation on medication errors during transition in the hospital setting. Pharm Pract. 2015;13(4):1-7. DOI: 10.18549/PharmPract.2015.04.634

4 - Ministério da Saúde (BR). Saúde da família: Uma estratégia para a reorientação do modelo assistencial. Brasília: Ministério da Saúde; 1997.

5 - Aljasmi F, Almalood F, Ansari A. Prevalence of medication errors in primary health care at Bahrain Defence Force Hospital: Prescription-based study. Drug Healthc Patient Saf. 2018;10:1-7. DOI: 10.2147/DHPS.S147994

6 - Poldervaart JM, van Melle AM, Willemse S, Wit NJ, Zwart DLM. In-hospital prescription changes and documentation in the medical records of the primary care provider: Results from a medical record review study. BMC Health Serv Res. 2017;17(792):1-10. DOI: 10.1186/s12913-017$\underline{2738-6}$

7 - Barberato LC, Scherer MDA, Lacourt RMC. O farmacêutico na atenção primária no Brasil: uma inserção em construção. Cienc Saúde Coletiva 2019;24(10):3717-26. DOI: 10.1590/1413$\underline{812320182410.30772017}$

8 - Vieira FS. Assistência farmacêutica no sistema público de saúde no Brasil. Rev Panam Salud Publica 2010 [citado em 20 mar 2020]; 27(2):149$56 . \quad$ Acesso em: https://scielosp.org/pdf/rpsp/v27n2/a10v27n2.pd f

9 - Nóbrega JF, Nitschke RG, Souza AIJ, Santos EKA. A sociologia compreensiva de Michel Maffesoli: Implicações para a pesquisa em enfermagem. Cogitare Enferm. 2012;17(2):373-6. DOI: 10.5380/ce.v17i2.24572

10 - Bardin L. Análise de conteúdo. 4. ed. Lisboa: Edições 70; 2008.

11 - World health organization (WHO). Universal health coverage: Everyone, everywhere. Geneva: WHO; 2018 [cited 2020 July 20]. Available in: http://www.who.int/campaigns/world-healthday/2018/World-Health-Day-2018-PolicyAdvocacy-Toolkit-Final.pdf?ua=1

12 - Footer K, Windle M, Ferguson L, Hatcher J, Lyons C, Gorin E, et al. A meta-narrative literature 
synthesis and framework to guide future evaluation of legal empowerment interventions. Health Hum Rights 2018 [cited 2020 July 25]; 20(2):65-84. Available in: https://www.ncbi.nlm.nih.gov/pmc/articles/PMC6 293352/pdf/hhr-20-065.pdf

13 - Garcia MM, Guerra Júnior AA, Acúrcio FA. Avaliação econômica dos programas Rede Farmácia de Minas do SUS versus Farmácia Popular do Brasil. Cienc Saúde Coletiva 2017;22(1):221-33. DOI: 10.1590/1413-81232017221.15912015

14 - Paniz VMV, Cechi ICCF, Fassa AG, Piccini RX, Tomassi E, Thumé E, et al. Acesso a medicamentos para tratamento de condições agudas prescritos a adultos nas regiões sul e nordeste do Brasil. Cad Saúde Pública 2016;32(4):1-13. DOI: $\underline{10.1590 / 0102-311 \times 00009915}$

15 - Miranda VIA, Fassa AG, Meucci RD, Lutz BH. Utilização do Programa Farmácia Popular do Brasil por idosos. Rev Saúde Pública 2016;50(13):1-13. DOI: $10.1590 /$ S1518-8787.2016050006180

16 - Costa JM, Carmo Junior NM, Pereira RCC, Crus $L F$, Figueiredo TP. Implantação de um serviço de contato telefônico pós alta hospitalar para acompanhamento farmacoterapêutico: Relato de experiência. Rev Bras Farm Hosp Serv Saúde 2015 [citado em 22 mar 2020]; 6(4):23-9. Acesso em: https://rbfhss.org.br/sbrafh/article/view/236/238

17 - Pellegrin K, Lee E, Reece U, Ayson C, Go R. Potentially preventable medication-related hospitalizations: A clinical pharmacist approach to assessment, categorization, and quality improvement. J Am Pharm Assoc. 2017;57:711-6. DOI: 10.1016/j.japh.2017.06.019

18 - Pérez-Jover V, Mira JJ, Carratala-Munuera C, Gil-Guillen VF, Basora J, López-Pineda A, et al. Inappropriate use of medication by elderly, polymedicated, or multipathological patients with chronic diseases. Int J Environ Res Public Health 2018;15(310):1-15. DOI: 10.3390/ijerph15020310

19 - Dwicaksono A, Fox AM. Does decentralization improve health system performance and outcomes in lowand middle-income countries? A systematic review of evidence from quantitative studies. Milbank Q 2018;96(2):323-68. DOI: 10.1111/1468$\underline{0009.12327}$
20 - Almeida PF, Giovanella L, Mendença MHM, Escorel S. Desafios à coordenação dos cuidados em saúde: estratégias de integração entre níveis assistenciais em grandes centros urbanos. Cad Saude Publica 2010;26(2):286-98. DOI: 10.1590/S0102-311X201000020000821

21 - Marques RM, Mendes A. Democracia, saúde pública e universalidade: O difícil caminhar. Saúde Soc 2007;16(3):35-51. DOI: 10.1590/S0104$\underline{12902007000300005}$

22 - Farris KB, Abramowitz PW, Weetman DB, Kaboli PJ, Dawson JD, James PA, et al. The lowa continuity of care study: Background and methods. Am J Health Syst Pharm. 2008;65(17):1631-42. DOI: $\underline{10.2146 / a j h p 070600}$

23 - Ni W, Colayco D, Hashimoto J, Komoto K, Gowda C, Wearda B, et al. Budget impact analysis of a pharmacist-provided transition of care program. J Manag Care Spec Pharm. 2018;24(2):90-6. DOI:

$\underline{10.18553 / \mathrm{imcp} .2018 .24 .2 .90}$

24 - Nazar H, Nazar Z, Portlock J, Todd A, Slight SP. A systematic review of the role of community pharmacies in improving the transition from secondary to primary care. Br J Clin Pharmacol. 2015;80(5):936-48. DOI: 10.1111/bcp.12718

25 - Kennelty KA, Chewning B, Wise M, Kind A, Roberts $T$, Kreling $D$. Barriers and facilitators of medication reconciliation processes for recently discharged patients from community pharmacists'perspectives. Res Social Adm Pharm. 2015;11(4):517-30.

DOI:

$\underline{10.1016 / j . s a p h a r m .2014 .10 .008}$

26 - Sharma N, O'Hare K, Antonelli RC, Sawicki GS. Transition care: Future directions in education, health policy, and outcomes research. Acad Pediatr. 2014;14(2):120-7. DOI:

$\underline{10.1016 / j . a c a p .2013 .11 .007}$

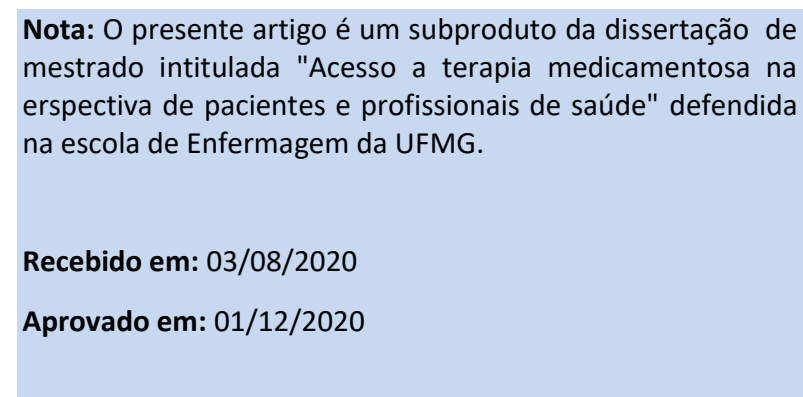

